\title{
THE NATURE OF FLAT SPECTRUM SOURCES
}

\author{
D.B. MELROSE \\ Research Centre for Theoretical Astrophysics \\ School of Physics, University of Sydney \\ NSW 2006 Australia
}

\begin{abstract}
Nonthermal radio sources near the Galactic Center with flat or weakly inverted spectra $\left(S(\omega) \propto \omega^{\alpha}\right.$ with $\left.\alpha \gtrsim 0\right)$ are attributed to optically thin synchrotron emission from a hard electron energy spectrum, $N(\varepsilon) \propto$ $\varepsilon^{-a}$ with $a=1-2 \alpha \lesssim 1$, produced by Fermi acceleration or diffusive shock acceleration at multiple shocks combined with a synchrotron pile up. This basic mechanism is also plausible for flat-spectrum AGN.
\end{abstract}

\section{Introduction}

Flat radio spectra, $S(\omega) \propto \omega^{\alpha}$ with $\alpha \gtrsim 0$, in some classes of AGN have been attributed to self absorption (e.g., Kellermann and Pauliny-Toth 1969). For the self-absorption peak to extend over at least a decade in frequency requires specific geometric properties in the source (e.g., Marscher 1977). This 'cosmic conspiracy' model (Cotton et al. 1980) is not consistent with interferometric data. Moreover, there are nonthermal sources with flat spectra in the Galactic center (GC) region (e.g., Yusef-Zadeh 1989) which are not plausibly self-absorbed. The simplest interpretation of these GC sources is in terms of optically thin synchrotron emission due to a hard electron spectrum, $N(\varepsilon)=K \varepsilon^{-a}$ with $a \lesssim 1$ (e.g., Anantharamaiah et al. 1991). Such an interpretation also needs to be considered for flat-spectrum AGN.

How can such hard electron spectra be produced? Diffusive shock acceleration (DSA) at a single strong shock cannot produce such a hard spectrum even when combined with synchrotron losses. It is suggested here that such hard spectra are produced through multiple DSA (DSA at multiple shocks), or Fermi-like acceleration, combined with synchrotron losses during the acceleration process. 


\section{Flat spectrum due self absorption}

The 'cosmic conspiracy' model for flat spectra requires that $S(\omega)$ depends on the size of the source, described by the radius $r$ in a conical model, only in the combination $\omega r$. The model requires a source with an area $A \propto r^{2}$, thickness $L \propto r$, a steady outflow implying $K \propto r^{-2}$ and an azimuthal magnetic field $B \propto r^{-1}$. The flat spectrum extends over the frequency range determined by the range of $r$ where the model is valid. The model implies that the linear size of the source is inversely proportional to frequency, which is not supported by interferometric data (Cotton et al. 1980).

\section{Flat spectrum sources near the Galactic center}

The flat spectrum sources in the GC region include linear filaments $30 \mathrm{pc} \times 1 \mathrm{pc}$ oriented nearly perpendicular to the Galactic plane, and threads $\sim 30 \mathrm{pc} \times 0.5 \mathrm{pc}$ (Yusef-Zadeh 1989). These sources are well removed (by tens of parsec) from the compact source $\mathrm{Sgr} \mathrm{A}^{*}$, which has some AGNlike properties. Spectral indices, measured for $\omega / 2 \pi=0.3-1.5 \mathrm{GHz}$ are in the range $\alpha \sim 0-0.3$. The magnetic field in these sources is relatively strong, $B \sim 10^{-3}-10^{-2} \mathrm{G}$, implying synchrotron lifetimes $\lesssim 10^{4} \mathrm{yr}$. These properties favor a model based on in situ acceleration due to shocks or large-scale turbulence (Anantharamaiah et al. 1991).

\section{Hardest electron spectra from acceleration alone}

Optically thin emission produces a flat spectrum, $\alpha \gtrsim 0$, for $a=1-2 \alpha \lesssim 1$. DSA at a single nonrelativistic shock produces a spectrum with $a=(r+$ $2) /(r-1)>2$ for a compression ratio $r<4$, implying $\alpha<-0.5$. A relativistic shock can produce only a slightly harder $(a>1.7)$ spectrum (Heavens and Drury 1989).

Both Fermi acceleration by MHD turbulence and DSA at many shocks are statistical in character and may be described in terms of diffusion in momentum space. DSA at many shocks implies systematic energy gains at each shock, and adiabatic losses between the shocks (e.g., Achterberg 1990; Schneider 1993). When synchrotron losses are included, the energy spectrum evolves according to

$$
\frac{\partial N(\varepsilon)}{\partial t}=\nu_{\mathrm{A}} \frac{\partial}{\partial \varepsilon}\left[\varepsilon^{4} \frac{\partial}{\partial \varepsilon} \frac{N(\varepsilon)}{\varepsilon^{2}}\right]-\frac{\partial}{\partial \varepsilon}\left[\dot{\varepsilon}_{\mathrm{syn}} N(\varepsilon)\right],
$$

where the Fermi acceleration and the synchrotron loss rates are given by

$$
\nu_{A} \sim \bar{\omega} \frac{v_{A}}{c}\left(\frac{\delta B}{B}\right)^{2}, \quad \dot{\varepsilon}_{\mathrm{syn}}=-\frac{4}{3} \sigma_{T} c W_{B}\left(\frac{\varepsilon}{m c^{2}}\right)^{2}
$$


respectively. Here $(\delta B / B)^{2}=W_{\text {turb }} / W_{B}$ is a measure of the level of the turbulence and $\bar{\omega}$ is its mean frequency, or is determined by the frequency with which shocks are encountered, $\sigma_{T}$ is the Thomson cross section, $v_{A}$ is the Alfvén speed and $W_{B}$ is the magnetic energy density.

In the absence of synchrotron losses, the asymptotic solution for injection at $\varepsilon=\varepsilon_{0}$ has $N(\varepsilon) \propto \varepsilon^{2}$ for $\varepsilon<\varepsilon_{0}$ and $N(\varepsilon) \propto \varepsilon^{-1}$ for $\varepsilon>\varepsilon_{0}$. Both analytic (Achterberg 1990; Schneider 1993) and numerical (Melrose and Pope 1993) calculations show that the asymptotic spectrum for DSA at multiple shocks is also $a=1$. Thus either Fermi acceleration or DSA at multiple shocks can account for a nearly flat synchrotron spectrum, $\alpha \lesssim 0$, but not for an inverted spectrum, $\alpha>0$.

\section{Synchrotron blocking}

Synchrotron losses combined with acceleration can lead to a pile up at $\varepsilon_{\mathrm{pu}}=\left|\dot{\varepsilon}_{\mathrm{syn}}\right| / \nu_{A}$, where the average energy gain due to acceleration balances the synchrotron loss. The pile up occurs only if the spectrum is already hard, $a<2$ (e.g., Melrose 1980, p. 110), and so is not possible for DSA at a single nonrelativistic shock, but is possible for Fermi acceleration or multiple DSA. The pile up hardens the spectrum toward $N(\varepsilon) \propto \varepsilon^{2}$ below $\varepsilon_{\mathrm{pu}}$. However, in a weak pile up the spectrum should be closer to $a=1$ than $a=-2$. The resulting synchrotron spectrum has $\alpha=(1-a) / 2$ for $a>2 / 3$ and $\alpha=1 / 3$ for $a<2 / 3$ below a peak frequency, $\omega_{\max }$, determined by the typical emission frequency of an electron at $\varepsilon_{\mathrm{pu}}$.

\section{Idealized Model}

Such a hard spectrum forms only when the acceleration is driven hard enough to cause a synchrotron pile up. A simple model for the maximum power input into relativistic particles involves an initial flux of Alfvén waves, e.g., due to a Kelvin-Helmohtz instability, with $\delta B \sim B$. A turbulent cascade transfers this energy to smaller scales where Fermi acceleration becomes effective (Bicknell and Melrose 1982). If synchrotron blocking is important, the power then goes directly into synchrotron emission due to electrons at $\sim \varepsilon_{\text {pu }}$. The following model incorporates these ideas. 1) The power input in turbulence or shocks is $\dot{W}_{\text {turb }}=\eta_{1}\left(v_{A} / L\right) W_{B}$ where $\eta_{1}=(\delta B / B)^{2}=W_{\text {turb }} / W_{B}$ is of order unity. 2) Synchrotron losses balance this power input, implying $\dot{W}_{\text {turb }}=\frac{4}{3} \sigma_{T} c W_{B} n_{\mathrm{pu}}\left(\varepsilon_{\mathrm{pu}} / m_{e} c^{2}\right)^{2}$, where $n_{\mathrm{pu}}$ is the number density of particles at $\sim \varepsilon_{\mathrm{pu}}$. 3) The pile up energy is related to the maximum frequency, $\varepsilon_{\mathrm{pu}} / m_{e} c^{2} \approx\left(\omega_{\max } / \Omega_{e}\right)^{1 / 2}$. 4) There is equipartition of energy, $n_{\mathrm{pu}} \varepsilon_{\mathrm{pu}}=\eta_{2} W_{B}$, with $\eta_{2}$ a constant of order unity.

For the GC filaments relevant input parameters are $\omega_{\max } / 2 \pi \sim 30 \mathrm{GHz}$, 
$L \sim 1 \mathrm{pc}$ and $B \sim 10^{-2} \mathrm{G}$. The model then requires $v_{A} / c \sim 10^{-2}$ which is plausible. The emission is optically thin as the model requires. For AGN a pile-up model was applied to infrared/optical emission by Schlickeiser (1984). This requires that $\omega_{\max } / 2 \pi \sim 10^{13} \mathrm{~Hz}$ be higher for AGN than for the GC sources. Such an AGN source would then be optically thick at radio frequencies and one needs to modify the model either by including multiple sources or by assuming a smooth $r$-dependence (cf. Marscher 1977). A smooth model is similar to the 'cosmic conspiracy' model, but differs from it in an important way. Provided that $S(\omega)$ increases with increasing $r$, optically thin emission dominates the observed emission and there is no implication that the source size should be a strong function of radio frequency.

\section{Conclusions}

The formation of hard electron spectra due to Fermi acceleration or multiple DSA coupled with synchrotron losses is plausible for flat or weakly inverted synchrotron spectra in the GC region and in AGN. The mechanism needs to be explored in more detail.

\section{References}

Achterberg, A. (1990) Particle acceleration by an ensemble of shocks, $A \& A$ 231, 251-258

Anantharamaiah, K.R., Pedlar, A., Ekers, R.D., and Goss, W.M. (1991) Radio studies of the Galactic centre - II. The arc, threads and related features at $90 \mathrm{~cm}(330 \mathrm{MHz})$, MNRAS, 249, 263-281

Bicknell, G.V., and Melrose, D.B. (1982) In situ acceleration in extragalactic radio jets, ApJ 262, 511-528

Cotton, W.D. et al. (1980) The very flat radio spectrum of 0735+178: A cosmic conspiracy?, $A p J, 238$, L123-L128

Heavens, A.F., and Drury, L.O'C. (1989) Relativistic shocks and particle acceleration, MNRAS, 235, 997-1009

Kellermann, K.I., and Pauliny-Toth, I.I.K. (1977) The spectra of opaque radio sources, ApJ 155, L71-L78

Marscher, A.P. (1977) Structure of radio sources with remarkably flat spectra: PKS $0735+178, A J, 82,781-784$

Melrose, D.B. (1980) Plasma Astrophysics Volume II Astrophysical Applications, Gordon \& Breach (New York)

Melrose, D.B., and Pope, M.H. (1993) Diffusive shock acceleration by multiple shocks, Proc. Astron. Soc. Australia, 10, 222-224

Schlickeiser, R. (1984) An explanation of abrupt cutoffs in the optical-infrared spectra of non-thermal sources. A new pile-up mechanism for relativistic electron spectra, $A \mathcal{E} A, 136,227-236$

Schneider, P. (1993) Diffusive particle acceleration by an ensemble of shock waves, $A \& A$ 278, 315-327

Yusef-Zadeh, F. (1989) Filamentary structures near the Galactic center, in The center of the Galaxy IAU Symp. 136, ed. Morris, M., D. Reidel (Dordrecht), pp. 243-263 